\title{
Genomic Copy Number Variations of the Complement Component C4B Gene Are Associated With Chronic Central Serous Chorioretinopathy
}

\author{
Myrte B. Breukink, ${ }^{1}$ Rosa L. Schellevis, ${ }^{1}$ Camiel J. F. Boon, ${ }^{2}$ Sascha Fauser, ${ }^{3}$ Carel B. Hoyng, ${ }^{1}$ \\ Anneke I. den Hollander, ${ }^{1,4}$ and Eiko K. de Jong ${ }^{1}$ \\ ${ }^{1}$ Department of Ophthalmology, Radboud University Medical Center, Nijmegen, the Netherlands \\ ${ }^{2}$ Department of Ophthalmology, Leiden University Medical Center, Leiden, the Netherlands \\ ${ }^{3}$ Department of Ophthalmology, University Hospital of Cologne, Cologne, Germany \\ ${ }^{4}$ Department of Human Genetics, Radboud University Medical Center, Nijmegen, the Netherlands
}

Correspondence: Eiko K. de Jong, Department of Ophthalmology, Radboud University Medical Center, Philips van Leydenlaan 15, 6525 EX Nijmegen, the Netherlands;

Eiko.deJong@radboudumc.nl.

MBB and RLS contributed equally to the work presented here and should therefore be regarded as equivalent authors.

Submitted: May 26, 2015

Accepted: July 23, 2015

Citation: Breukink MB, Schellevis RL, Boon CJF, et al. Genomic copy number variations of the complement component $C 4 B$ gene are associated with chronic central serous chorioretinopathy. Invest Ophthalmol Vis Sci. 2015;56:5608-5613. DOI:10.1167/ iovs.15-17343
Purpose. Chronic central serous chorioretinopathy (CCSC) has recently been associated to variants in the complement factor $\mathrm{H}$ gene. To further investigate the role of the complement system in cCSC, the genomic copy number variations in the complement component 4 gene (C4) were studied.

Methods. $C 4 A$ and $C 4 B$ copy numbers were analyzed in 197 cCSC patients and 303 healthy controls by using a Taqman copy number determination assay. Copy numbers of $C 4 A, C 4 B$, and the total $C 4$ load were compared between cases and controls, by using a Fisher exact test. For this analysis Bonferroni correction was performed for three tests, and $P$ values $<0.017$ were considered to be significant. A logistic regression model was constructed to calculate the odds ratios (ORs) of each of the $C 4 B$ copy numbers, using two copies as a reference. For this model $P$ values $<0.05$ were considered to be significant.

RESults. $C 4 B$ genomic copy numbers differed significantly between cCSC patients and healthy controls $(P=0.0018)$. Absence of $C 4 B$ significantly conferred risk of cCSC $(P=0.039, \mathrm{OR}=$ $2.61[95 \%$ confidence interval $(C D)=1.05-6.52]$ ), whereas three copies of $C 4 B$ significantly decreased the risk of $\operatorname{cCSC}(P=0.014, \mathrm{OR}=0.45[95 \% \mathrm{CI}=0.23-0.85])$. The $C 4 A$ genomic copy numbers and total $C 4$ load did not significantly differ between cases and controls.

Conclusions. This study showed that copy numbers of $C 4 B$ are significantly associated with cCSC. Carrying no copies of $C 4 B$ significantly increases the risk of cCSC, whereas carrying three $C 4 B$ copies is protective. These findings reinforce the hypothesis of a possible involvement of the complement system in the pathogenesis of cCSC.

Keywords: chronic central serous chorioretinopathy, cCSC, complement component 4, C4, $C 4 A, C 4 B$

\begin{abstract}
Chronic central serous chorioretinopathy (cCSC) is characterized by fluid accumulation under the neuroretina. It has been postulated that this serous fluid derives from the choroid and that it leaks through a dysfunctional retinal pigment epithelium, causing a detachment of the neuroretina. ${ }^{1-4}$ Classically, cCSC patients are relatively young (middle-aged) men who are still professionally active. ${ }^{5}$ Besides male sex, also the use of corticosteroids, type A personality, and stress have been associated with cCSC. ${ }^{4-6}$ Although the exact pathophysiological mechanism of the disease remains unknown, we and others have previously suggested involvement of the complement system, and in particular the complement factor $\mathrm{H}$ gene $(\mathrm{CFH})$ in cCSC. ${ }^{7,8}$ Interestingly, $\mathrm{CFH}$ variants that confer increased risk in cCSC have previously been described to be protective in age-related macular degeneration, and vice versa. ${ }^{8-10}$
\end{abstract}

The complement system consists of three major pathways: the classical, the lectin, and the alternative pathway. ${ }^{11} \mathrm{CFH}$ is involved in the alternative pathway and can influence $\mathrm{C} 3 \mathrm{~b}$ production by blocking one of the two $\mathrm{C} 3$-convertases
$(\mathrm{C} 3 \mathrm{bBb})$. The classical and lectin pathways also play an important role in $\mathrm{C} 3 \mathrm{~b}$ production mediated by the other $\mathrm{C} 3$ convertase (C4b2a), of which the complement component 4 (C4) protein is a key factor. ${ }^{12}$

Copy number variations of the $C 4$ gene have been associated with several eye diseases and autoimmune disorders (e.g., Vogt-Koyanagi-Harada and Behçet's disease). ${ }^{13-15}$ In addition, Banlaki et al. ${ }^{16}$ have found that the genomic copy number of $C 4 B$ is associated with cortisol release after adrenocorticotropic hormone (ACTH) stimulation. This is of particular interest considering that stress, which appears to be associated with $\operatorname{cCSC},{ }^{4}$ has a strong influence on the hypothalamic-pituitary-adrenal (HPA) axis and increases ACTH release. ${ }^{17}$

Because of its role in the complement system, and its association with the HPA axis, we hypothesized that copy number variation in the $C 4$ gene may be associated with cCSC. In this study we assessed the copy number variations of the $C 4$ gene in a cCSC cohort. 
Table 1. Demographics of the Study Population

\begin{tabular}{lccc}
\hline & cCSC Patients & Controls & P Value \\
\hline No. of subjects & 197 & 303 & NA \\
Sex, male/female & $154 / 43$ & $226 / 77$ & 0.392 \\
Age, mean \pm SD, y & $53 \pm 10$ & $53 \pm 11$ & 0.755 \\
Age range, y & $29-74$ & $29-77$ & NA \\
\hline
\end{tabular}

NA, not annotated; SD, standard deviation.

\section{Materials ANd Methods}

\section{Subjects}

In this study, 197 patients diagnosed with cCSC who visited the outpatient clinic of the Department of Ophthalmology at the Radboud University Medical Center, Nijmegen, the Netherlands, were included (Table 1). The diagnosis cCSC was based on an extensive ophthalmologic examination including fundoscopy, spectral-domain optical coherence tomography, fluorescein angiography, and indocyanine green angiography. The definition of typical cCSC used in this study was based on the previously published subgroups by de Jong et al., ${ }^{8}$ and patients in this study underwent phenotyping by an experienced retina specialist (CJFB) (Fig. 1A-F). Additionally, a total of 303 control subjects were recruited from the blood bank of the Radboud University Medical Center $(n=154)$ and the European Genetic Database (EUGENDA, www.eugenda.org; provided in the public domain by the University Hospital of Cologne, Cologne, Germany and the Radboud University Medical Center) $(n=149)$ (Table 1$)$. For this last group, fundus photographs were graded to rule out any ophthalmologic abnormalities at the moment of inclusion. Informed consent for the use of DNA for genetic studies was obtained from all subjects. This study followed the guidelines of the Declaration of Helsinki and was approved by the local ethics committee.

\section{Copy Number Determination}

DNA was isolated from peripheral blood by using standard procedures. $C 4 A$ and $C 4 B$ copy numbers were determined by real-time PCR using Taqman genotyping assays (Applied Biosystems, Thermo Fisher Scientific, Waltham, MA, USA). The FAM-labeled $C 4 A$ (Hs07226349_cn) or $C 4 B$ (Hs07226350_cn) Taqman copy number assay was combined with the VIC-labeled Ribonuclease P (RNaseP) reference assay (catalog No. 4403326), and Taqman genotyping mastermix (catalog No. 4381656). All samples were tested in duplicate for
$C 4 A$ and $C 4 B$ on 384-wells plates by using 10 ng DNA in a total reaction volume of $10 \mu \mathrm{L}$.

Samples with known copy numbers for either $C 4 A(0-4)$ or $C 4 B(0-3)$ were kindly provided by C. Yung Yu. ${ }^{18}$ These samples were included as a reference on each plate to facilitate accurate copy number determination, using the method described previously. ${ }^{18}$ In each run the amplification efficiencies of the $C 4 A / C 4 B$ and $R N a s e P$ probes were calculated by using a serial dilution (50-1.56 ng) of a sample with two $C 4 A$ and $C 4 B$ copies. The primer efficiencies of the probes were compared and deemed similar if they differed $<2 \%$; this was the case in all runs. Therefore, the efficiencies were not incorporated into the calculations of the copy numbers. Polymerase chain reaction was performed with a 7900HT thermocycler (Applied Biosystems, Thermo Fisher Scientific) using the following program: 2 minutes at $50^{\circ} \mathrm{C}, 10$ minutes at $95^{\circ} \mathrm{C}$, and 40 cycles of 15 seconds at $95^{\circ} \mathrm{C}$ and 60 seconds at $60^{\circ} \mathrm{C}$. Data were analyzed with the Copycaller software (V2.0; Applied Biosystems, Thermo Fisher Scientific). Copy numbers determined by the Copycaller software were corrected by using the trend line based on the reference samples, as described before. ${ }^{18}$ If the results were inconsistent between the Copycaller output and the values corrected with the trend line, samples were retested on a new plate in triplicate.

\section{Statistics}

The comparison of $C 4 A, C 4 B$, and total $C 4$ copy number distribution between cCSC patients and controls was performed by means of a Fisher exact test using SPSS Statistics (V20; IBM Corp., Armonk, NY, USA). Bonferroni correction for multiple testing was performed for three tests and $P$ values $<$ 0.017 were considered to be statistically significant. A logistic regression model was constructed to determine the odds ratios (ORs) for the various copy numbers of $C 4 B$. According to previously published studies, two genomic copy numbers of $C 4 B$ are considered to be most common in the healthy population. ${ }^{15,19}$ We were able to confirm this in our cohort, and therefore this copy number was set as reference. In this model, $P$ values $<0.05$ were considered to be significant. Graphs were generated by using Graphpad Prism (V5; Graphpad Software, San Diego, CA, USA).

\section{Results}

The copy numbers of $C 4 A$ and $C 4 B$ were successfully determined in 197 cCSC cases and 303 controls. No significant difference was observed between cases and controls for the $C 4 A$ genomic copy number (range: $0-6, P=0.649$; Fig. $2 A$ ).
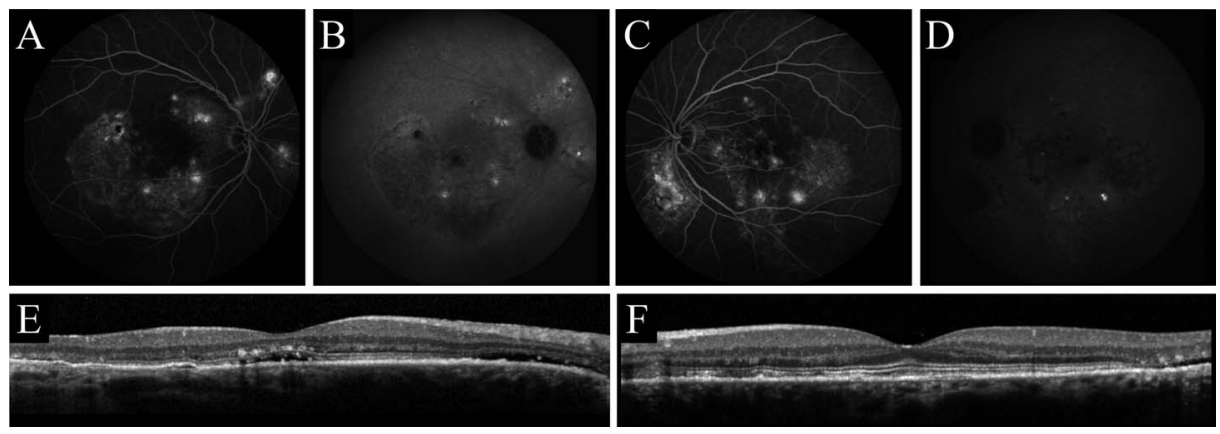

Figure 1. Example of FA of an RE (A) and an LE (C), ICG angiography of an RE (B) and an LE (D), and spectral-domain optical coherence tomography of an RE (E) and an LE (F) imaging of the phenotypic characteristics of the CCSC cohort used for this study. (A-F) The RE and LE of a patient demonstrate diffuse hyperfluorescent areas of leakage on FA and ICG angiography (A-D) and subretinal fluid beneath the fovea (E), illustrative for typical cCSC. FA, fluorescein angiography; ICG, indocyanine green; LE, left eye; RE, right eye. 
A

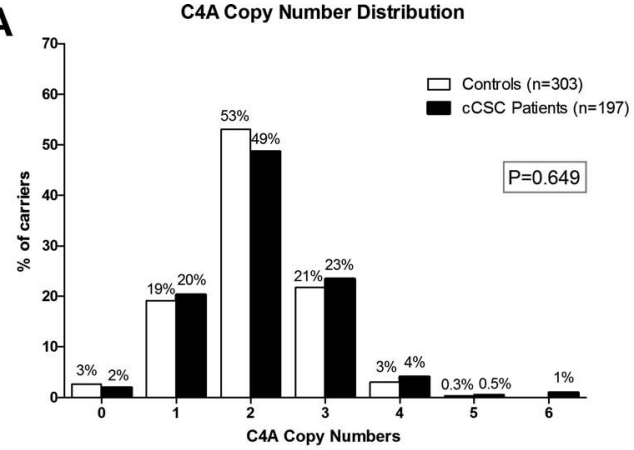

C

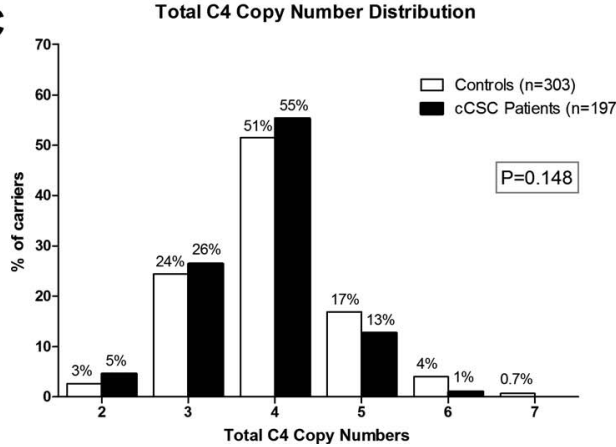

B

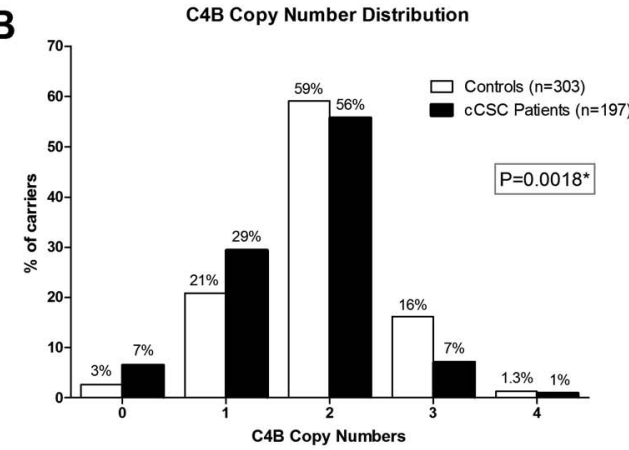

Figure 2. Distribution of the genomic copy numbers of $C 4 A(\mathbf{A}), C 4 B(\mathbf{B})$, and total $C 4$ (C) in patients with cCSC and controls. Displayed $P$ values were generated with a Fisher exact test. To correct for multiple testing, $P$ values $<0.017$ were considered to be significant.

The $C 4 B$ distribution was significantly different between cCSC patients and controls (range: $0-4, P=0.0018$; Fig. 2B). Overall, cases carried lower copy numbers of $C 4 B$ than the control population. The total $C 4$ genomic copy number was not different in cases compared to controls ( $P=0.148$; Fig. 2C). Age and sex were not associated with $C 4 A, C 4 B$, or total $C 4$ genomic copy number (Table 2 ; data for $C 4 A$ and total $C 4$ not shown).

To assess the effect size of the different copy numbers of $C 4 B$ on development of cCSC, a logistic regression was performed (Table 2). The logistic regression model based on the distribution of $C 4 B$ between cases and controls was significant $(P=0.0035$; Table 2$)$. Carrying no copies of $C 4 B$ conferred increased risk of $\mathrm{cCSC}(P=0.039, \mathrm{OR}=2.61,95 \%$ confidence interval $[\mathrm{CI}]=1.05-6.52)$. A similar trend was observed for carriers of one copy of $C 4 \mathrm{~B}$, but the results were not significant $(P=0.080, \mathrm{OR}=1.47,95 \% \mathrm{CI}=0.96-2.26)$. Carrying three $C 4 B$ copies was associated with a significantly decreased risk of $\operatorname{cCSC}(P=0.014, \mathrm{OR}=0.45,95 \% \mathrm{CI}=0.24-$ $0.85)$, whereas no significant association with CCSC was observed in individuals carrying four copies of $C 4 B(P=0.81)$.

\section{Discussion}

Our study results demonstrated that cCSC patients have a significantly different $C 4 B$ load as compared to healthy controls $(P=0.0018)$. Carrying no copies of $C 4 B$ was associated with an increased risk of cCSC $(\mathrm{OR}=2.61,95 \% \mathrm{CI}$ $=1.05-6.52)$, whereas carrying three $C 4 B$ copies was associated with a decreased risk of $\mathrm{cCSC}(\mathrm{OR}=0.45,95 \% \mathrm{CI}$ $=0.23-0.85)$. No association with cCSC was observed in individuals carrying four $C 4 B$ copies, which is likely due to the limited sample size of this group (cases, $n=4$; controls, $n=2$ ). No significant differences were observed between cases and controls for $C 4 A$ and total $C 4$ load.
The $C 4$ gene lies within the $\underline{R} P-\underline{C} 4-\underline{C} Y P 21-T N \underline{X}$ (RCCX) locus located in the major histocompatability complex (MHC) region III on chromosome 6 of the human genome. ${ }^{19}$ The MHC region contains an elevated level of genomic copy number variations that are presumably present to increase immunologic diversity. ${ }^{20}$ Duplications and deletions in the region have led to the formation of haplotypes containing variable copies of the $R C C X$ locus in the human population (Fig. 3B).$^{20-22}$ Haplotypes containing two or more duplications of the RCCX locus show extensive variability in their gene content, generally with complete duplications of the $C 4$ gene (Fig. 3B). ${ }^{21,23}$ The $C 4$ gene encodes the $\mathrm{C} 4$ protein, of which two variants have been described $(\mathrm{C} 4 \mathrm{~A} / \mathrm{C} 4 \mathrm{~B})$, differing in only four amino acids encoded by exon 26 (Fig. 3A). ${ }^{24}$

Copy number variations of either $C 4 A$ or $C 4 B$ have been associated with several systemic diseases with ocular involvement, such as Vogt-Koyanagi-Harada disease, Behçet's disease, and systemic lupus erythematosus (SLE). ${ }^{13-15}$ Hou et al. ${ }^{13}$ have shown that a lower copy number of $C 4 A$ and $C 4 B$ increases the risk of Vogt-Koyanagi-Harada disease, an autoimmune disorder characterized by bilateral granulomatous panuveitis. The same group ${ }^{14}$ has also demonstrated that higher copy numbers of $C 4 A$ confer risk of Behçet's disease, an autoinflammatory disease, which presents with acute anterior uveitis. Several studies $^{15,25,26}$ have shown an association between low copy numbers of $C 4$ and an increased risk for SLE, an autoimmune disease that is typically mediated by immune complexes. In the past, CSC has been described in SLE patients, ${ }^{27,28}$ but it remains unclear whether this is a primary manifestation of SLE, or whether it is a consequence of corticosteroid treatment for SLE. ${ }^{29,30}$ Several studies have reported a positive linear correlation between serum $\mathrm{C} 4$ and $C 4$ genomic copy number, ${ }^{31-33}$ suggesting that the lower number of $C 4 B$ copies in cCSC patients leads to lower systemic C4B levels. This may indicate that an overall lower activity of the complement system might be present in CCSC patients. 
Table 2. Logistic Regression Model for $C 4 B$ Load

\begin{tabular}{lccccc}
\hline \multicolumn{7}{c}{ Overall Significance Model, $\boldsymbol{P}=\mathbf{0 . 0 0 3 5}$} \\
\hline $\begin{array}{l}\text { C4B Copy } \\
\text { Number }\end{array}$ & $\begin{array}{c}\text { Patients, } \\
\text { C Controls, }\end{array}$ & & & \\
\hline Age & $\boldsymbol{n}$ & $\boldsymbol{n}$ & $\boldsymbol{P}$ Value & OR & 95\% CI \\
Sex & 197 & 303 & 0.605 & NA & NA \\
0 & 197 & 303 & 0.346 & NA & NA \\
1 & 13 & 8 & 0.039 & 2.613 & $1.048-6.518$ \\
2 & 58 & 63 & 0.080 & 1.469 & $0.956-2.259$ \\
3 & 110 & 179 & Ref & 1 & NA \\
4 & 14 & 49 & 0.014 & 0.445 & $0.234-0.849$ \\
& 2 & 4 & 0.808 & 0.809 & $0.145-4.503$
\end{tabular}

Ref, reference.

Recently, low copy numbers of $C 4 B$ have been associated with hyperreactivity of the HPA axis. ${ }^{16}$ Banlaki et al. ${ }^{16}$ have shown that in patients with adrenal incidentaloma and low $(<2$ copies) $C 4 B$ genomic copy number, baseline ACTH is significantly reduced as compared to high ( $\geq 2$ copies) genomic copy number of $C 4 B$. Moreover, a significantly higher cortisol response is observed after ACTH stimulation in the patients with low $C 4 B$ genomic copy number. ${ }^{16}$

These results are of interest in the context of cCSC because of the described clinical associations with stress and the use of corticosteroids that both exert physiological effects at the level of the HPA axis. ${ }^{17}$ Various relatively small studies have studied cortisol levels in cCSC patients. Although 24-hour urine samples show elevated cortisol levels in cCSC patients in certain studies, ${ }^{34,35}$ these results are not observed in single serum measurements during set times in other studies. ${ }^{36,37}$ These discrepancies could be explained by variable cortisol fluctuations between individuals during the day, and therefore changes in endogenous cortisol levels cannot be ruled out as a hallmark of cCSC. It is possible that patients with cCSC generally have normal cortisol levels but respond differently to stimulation of the HPA axis. Stress, which also appears to be associated with cCSC,, 38 stimulates the HPA axis and could lead to temporarily elevated cortisol levels in patients as compared to healthy individuals. How high levels of cortisol can lead to subretinal fluid accumulation is currently unknown. A study in rats suggests that the disease mechanism could be mediated by binding of corticosteroids to the mineralocorticoid receptor. ${ }^{39}$ In this study, activation of the mineralocorticoid receptor causes vascular effects similar to those observed in $\operatorname{cCSC},{ }^{39}$ but the underlying pathways still remain to be elucidated.

The mechanism through which low copy numbers of $C 4 B$ may lead to hyperresponsiveness of the HPA axis is unclear. It has been hypothesized that it is not the $C 4 B$ gene, but rather the neighboring CYP21A2 gene, that mediates this effect. ${ }^{16}$ The CYP21A2 gene encodes the enzyme 21-hydroxylase, which plays an important role in the steroid metabolism pathway by converting progesterone and 17- $\alpha$-hydroxyprogesterone to 11-deoxycorticosterone and 11-deoxycortisol, respectively. Because of the genomic structure of the $R C C X$ locus, variation in the CYP21A2 gene is in high linkage disequilibrium with variation in the neighboring $C 4$ gene $(C 4 A$ or $C 4 B) .{ }^{16}$ Therefore, further exploration of the precise structure and specific variations present in the $R C C X$ locus may reveal new insights into the pathogenesis of CCSC.

The current study and previous studies identified an association between complement genes and $\operatorname{cSC},{ }^{7,8}$ suggesting that the complement system may be dysregulated in cCSC. Taken together, these findings may indicate that the immune system, influenced by environmental factors such as stress, could play a pivotal role in the pathophysiology of CCSC. Further studies are necessary to determine the physiological effects of genetic variation at the $C 4$ gene and the RCCX locus in CCSC.

\section{Acknowledgments}

We thank C. Yung Yu, DPhil, for providing the positive control samples (Center for Molecular and Human Genetics, The Research Institute, City, Ohio, USA). We thank Joannes M. M. Groenewoud

A.

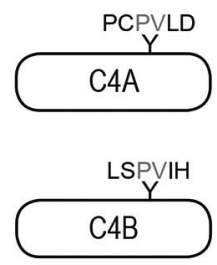

B.

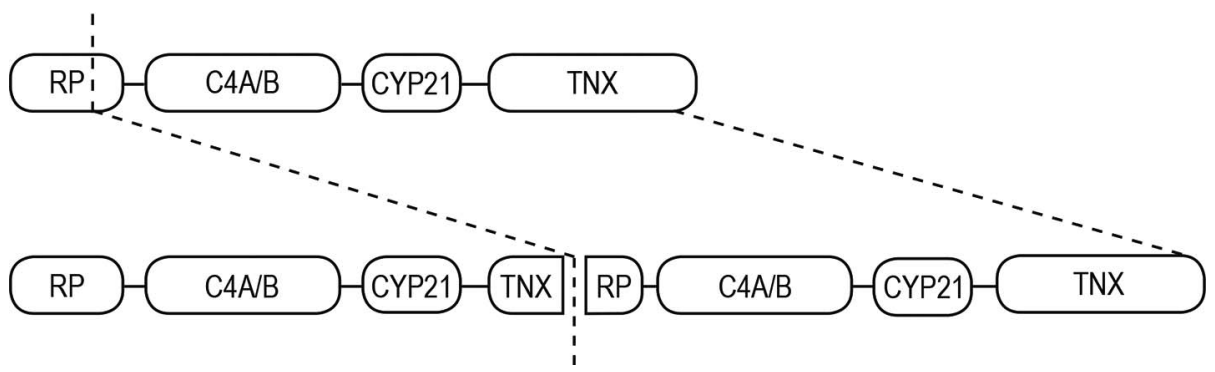

Figure 3. A schematic illustration of the $R C C X$ locus. (A) A display of the two variants of the $C 4$ gene, differing in four amino acids in exon 26 (p.1101-1106). (B) An example of the structure of the $R C C X$ locus with a single copy (upper panel) and two copies (lower panel) of the C4 gene. In case of duplication, a complete $C 4 A$ or $C 4 B$ gene and the $C Y P 21$ gene are duplicated, while the $R P$ and $T N X$ genes are only partially duplicated. The dotted lines indicate the approximate site where during recombination the duplication has occurred in the past (Figure based on Banlaki et al. 2013)..$^{22}$ 
(MSc) for his statistical support (Department of Health Evidence, Radboudumc, Nijmegen, the Netherlands).

Supported by the Macula Vision Research Foundation, MD Fonds, Landelijke Stichting voor Blinden en Slechtzienden, Gelderse Blindenstichting, Stichting Nederlands Oogheelkundig Onderzoek, Stichting Blindenhulp, Stichting A.F. Deutman Oogheelkunde Researchfonds, Nijmeegse Oogonderzoek Stichting, Janivo Stichting, Retina Netherlands, Stichting Blinden Penning, Oogfonds, Gisela Thier Fellowship of Leiden University, and the Radboud Institute for Molecular Life Sciences. The authors alone are responsible for the content and writing of the paper.

Disclosure: M.B. Breukink, None; R.L. Schellevis, None; C.J.F. Boon, None; S. Fauser, None; C.B. Hoyng, None; A.I. den Hollander, None; E.K. de Jong, None

\section{References}

1. Wang M, Munch IC, Hasler PW, Prunte C, Larsen M. Central serous chorioretinopathy. Acta Ophthalmol. 2008;86:126145.

2. Gemenetzi M, De Salvo G, Lotery AJ. Central serous chorioretinopathy: an update on pathogenesis and treatment. Eye (Lond). 2010;24:1743-1756.

3. Nicholson B, Noble J, Forooghian F, Meyerle C. Central serous chorioretinopathy: update on pathophysiology and treatment. Surv Ophthalmol. 2013;58:103-126.

4. Liew G, Quin G, Gillies M, Fraser-Bell S. Central serous chorioretinopathy: a review of epidemiology and pathophysiology. Clin Experiment Ophthalmol. 2013;41:201-214.

5. Yannuzzi LA. Central serous chorioretinopathy: a personal perspective. Am J Ophthalmol. 2010;149:361-363.

6. Bouzas EA, Karadimas P, Pournaras CJ. Central serous chorioretinopathy and glucocorticoids. Surv Ophthalmol. 2002; $47: 431-448$

7. Miki A, Kondo N, Yanagisawa S, Bessho H, Honda S, Negi A. Common variants in the complement factor $\mathrm{H}$ gene confer genetic susceptibility to central serous chorioretinopathy. Ophthalmology. 2014;121:1067-1072.

8. de Jong EK, Breukink MB, Schellevis RL, et al. Chronic central serous chorioretinopathy is associated with genetic variants implicated in age-related macular degeneration. Ophthalmology. 2015;122:562-570.

9. Hageman GS, Anderson DH, Johnson LV, et al. A common haplotype in the complement regulatory gene factor $\mathrm{H}$ (HF1/ $\mathrm{CFH})$ predisposes individuals to age-related macular degeneration. Proc Natl Acad Sci U S A. 2005;102:7227-7232.

10. Fritsche LG, Chen W, Schu M, et al. Seven new loci associated with age-related macular degeneration. Nat Genet. 2013;45: 433-439.

11. Anderson DH, Radeke MJ, Gallo NB, et al. The pivotal role of the complement system in aging and age-related macular degeneration: hypothesis re-visited. Prog Retin Eye Res. 2010; 29:95-112.

12. Ricklin D, Hajishengallis G, Yang K, Lambris JD. Complement: a key system for immune surveillance and homeostasis. Nat Immunol. 2010;11:785-797.

13. Hou S, Qi J, Liao D, et al. High C4 gene copy numbers protects against Vogt-Koyanagi-Harada syndrome in Chinese Han. Br J Ophthalmol. 2014;98:1733-1737.

14. Hou S, Qi J, Liao D, et al. Copy number variations of complement component $\mathrm{C} 4$ are associated with Behcet's disease but not with ankylosing spondylitis associated with acute anterior uveitis. Arthritis Rheum. 2013;65:2963-2970.

15. Yang Y, Chung EK, Wu YL, et al. Gene copy-number variation and associated polymorphisms of complement component $\mathrm{C} 4$ in human systemic lupus erythematosus (SLE): low copy number is a risk factor for and high copy number is a protective factor against SLE susceptibility in European Americans. Am J Hum Genet. 2007;80:1037-1054.

16. Banlaki Z, Raizer G, Acs B, et al. ACTH-induced cortisol release is related to the copy number of the $\mathrm{C} 4 \mathrm{~B}$ gene encoding the fourth component of complement in patients with nonfunctional adrenal incidentaloma. Clin Endocrinol (Oxf). 2012;76:478-484

17. Aguilera G. Regulation of pituitary ACTH secretion during chronic stress. Front Neuroendocrinol. 1994;15:321-350.

18. Wu YL, Savelli SL, Yang Y, et al. Sensitive and specific real-time polymerase chain reaction assays to accurately determine copy number variations (CNVs) of human complement C4A, C4B, C4-long, C4-short, and RCCX modules: elucidation of C4 CNVs in 50 consanguineous subjects with defined HLA genotypes. J Immunol. 2007;179:3012-3025.

19. Szilagyi A, Fust G. Diseases associated with the low copy number of the $\mathrm{C} 4 \mathrm{~B}$ gene encoding $\mathrm{C} 4$, the fourth component of complement. Cytogenet Genome Res. 2008;123:118-130.

20. Olsson LM, Holmdahl R. Copy number variation in autoimmunity-importance hidden in complexity? Eur J Immunol. 2012;42:1969-1976.

21. Chung EK, Yang Y, Rennebohm RM, et al. Genetic sophistication of human complement components $\mathrm{C} 4 \mathrm{~A}$ and $\mathrm{C} 4 \mathrm{~B}$ and RP-C4-CYP21-TNX (RCCX) modules in the major histocompatibility complex. Am J Hum Genet. 2002;71:823-837.

22. Banlaki Z, Szabo JA, Szilagyi A, et al. Intraspecific evolution of human RCCX copy number variation traced by haplotypes of the CYP21A2 gene. Genome Biol Evol. 2013;5:98-112.

23. Blanchong CA, Zhou B, Rupert KL, et al. Deficiencies of human complement component $\mathrm{C} 4 \mathrm{~A}$ and $\mathrm{C} 4 \mathrm{~B}$ and heterozygosity in length variants of RP-C4-CYP21-TNX (RCCX) modules in Caucasians: the load of RCCX genetic diversity on major histocompatibility complex-associated disease. J Exp Med. 2000;191:2183-2196.

24. Yu CY, Belt KT, Giles CM, Campbell RD, Porter RR. Structural basis of the polymorphism of human complement components $\mathrm{C} 4 \mathrm{~A}$ and $\mathrm{C} 4 \mathrm{~B}$ : gene size, reactivity and antigenicity. EMBO J. 1986;5:2873-2881.

25. Lv Y, He S, Zhang Z, et al. Confirmation of C4 gene copy number variation and the association with systemic lupus erythematosus in Chinese Han population. Rbeumatol Int. 2012;32:3047-3053.

26. Ptacek T, Li X, Kelley JM, Edberg JC. Copy number variants in genetic susceptibility and severity of systemic lupus erythematosus. Cytogenet Genome Res. 2008;123:142-147.

27. Eckstein MB, Spalton DJ, Holder G. Visual loss from central serous retinopathy in systemic lupus erythematosus. $\mathrm{Br} \mathrm{J}$ Ophthalmol. 1993;77:607-609.

28. Cunningham ET Jr, Alfred PR, Irvine AR. Central serous chorioretinopathy in patients with systemic lupus erythematosus. Ophthalmology. 1996;103:2081-2090.

29. Bouzas E, Mastorakos G. Central serous retinopathy in systemic lupus erythematosus: a manifestation of the disease or of its treatment? Br J Ophthalmol. 1994;78:420-421.

30. Bouyon A, Costedoat-Chalumeau N, Limal N, et al. Central serous chorioretinopathy and systemic diseases report of 2 cases associated with corticotherapy [in French]. Rev Med Interne. 2006;27:487-491.

31. Margery-Muir AA, Wetherall JD, Castley AS, et al. Establishment of gene copy number-specific normal ranges for serum $\mathrm{C} 4$ and its utility for interpretation in patients with chronically low serum C4 concentrations. Arthritis Rbeumatol. 2014;66: 2512-2520.

32. Uko G, Christiansen FT, Dawkins RL, McCann VJ. Reference ranges for serum $\mathrm{C} 4$ concentrations in subjects with and without C4 null alleles. J Clin Pathol. 1986;39:573-576.

33. Yang Y, Chung EK, Zhou B, et al. Diversity in intrinsic strengths of the human complement system: serum $\mathrm{C} 4$ protein 
concentrations correlate with $\mathrm{C} 4$ gene size and polygenic variations, hemolytic activities, and body mass index. $J$ Immunol. 2003;171:2734-2745.

34. Haimovici R, Rumelt S, Melby J. Endocrine abnormalities in patients with central serous chorioretinopathy. Ophthalmology. 2003;110:698-703.

35. Shang Q, Liu C, Wei S, Shi F, Li Y, Qiao L. Determination of cortisol in plasma and 24-hour urine of patients with central serous chorioretinopathy [in Chinese]. Zhonghua Yan Ke Za Zhi. 1999;35:297-299.
36. Tufan HA, Gencer B, Comez AT. Serum cortisol and testosterone levels in chronic central serous chorioretinopathy. Graefes Arch Clin Exp Ophthalmol. 2013;251:677-680.

37. Zakir SM, Shukla M, Simi ZU, Ahmad J, Sajid M. Serum cortisol and testosterone levels in idiopathic central serous chorioretinopathy. Indian J Ophthalmol. 2009;57:419-422.

38. Yannuzzi LA. Type A behavior and central serous chorioretinopathy. Trans Am Ophthalmol Soc. 1986;84:799-845.

39. Zhao M, Celerier I, Bousquet E, et al. Mineralocorticoid receptor is involved in rat and human ocular chorioretinopathy. J Clin Invest. 2012;122:2672-2679. 